\title{
HACIA LA MINIATURIZACIÓN DE LA PRENSA FEMENINA. EL CASO GLAMOUR'
}

\author{
María Ganzabal Learreta \\ (Universidad del País Vasco) \\ maria.ganzabal@ehu.es
}

\section{Resumen:}

Las revistas de pequeño formato no son un concepto nuevo. Están presentes desde hace mucho tiempo en el universo de las revistas de prensa: tal es el caso de National Geographic (1889), Science et Vie (1913), Reader's Digest (1922), TV Guide (1953), Teleprograma (1966), Télé Poche (1966) o Télé Z (1973). Pero es a partir de 1998 con el lanzamiento en Italia de Glamour cuando este pequeño formato se consolida como el formato de éxito y de futuro para la prensa femenina. Ahora, el formato de moda en la prensa femenina es el "mini", "pocket" (de bolsillo), o "handbag-size" (tamaño para el bolso); la denominación difiere según el país de que se trate.

Palabras clave: Revista, formato, bolsillo, tamaño, mujer.

\begin{abstract}
:
Small format magazines are not a new concept. They have been present in the universe of magazines for a long time, as is the case with National Geographic (1889), Science et Vie (1913), Reader's Digest (1922), TV Guide (1953), Teleprograma (1966), Télé Poche (1966) or Télé Z (1973), but it has been since 1998, with the launch in Italy of Glamour, that this small format has become consolidated as the successful format of the future for women's magazines. In the publishing sector, small has become the big thing in magazines. Now, the fashionable format for women's magazines is the "mini", "pocket" or "handbag-size"; the name changes depending on the country.
\end{abstract}

Key words: Magazine, format, pocket, size, women.

\footnotetext{
${ }^{1}$ Este artículo se basa parcialmente en algunas entrevistas realizadas a grandes profesionales de los principales grupos editoriales como Oscar Becerra, director de Marketing y Expansión del Grupo Zeta, Juan Elvira y María Isabel Abad, responsables del Departamento de Marketing de Cosmopolitan, Mónica Martínez, directora de Marketing Editorial de Woman, Samari Fernández Feito del Departamento de Marketing de Telva, Belén Ceda, responsable de Maquetación de Marie Claire y María José Martín, responsable de Marketing de Hachette Filipacchi. Vaya desde aquí mi agradecimiento a todos ellos.
} 


\section{SMALL IS BEAUTIFULL. CARACTERÍSTICAS DE LAS REVISTAS MINI, POC- KET O DE BOLSILLO}

\subsection{El tamaño}

La primera característica diferencial de este nuevo formato de prensa es su pequeño tamaño. Se trata, en la mayoría de los casos, de una reducción proporcional del 70\% (también llamado A5) de la página originaria, que es un A4. El formato pequeño plantea problemas de maqueta, qué duda cabe. Hay que dar casi la misma información pero sin recargar las páginas. No basta con reducir las maquetas. Hay que maquetar de una manera diferente y elegir cuerpos tipográficos más legibles. Cuando se hacen dos ediciones (grande y pequeña) al mismo tiempo, se debe hacer una reducción proporcional de la página para no multiplicar por dos la carga de trabajo de los maquetistas. En este último caso, el tamaño del cuerpo se debe agrandar en la edición de formato grande. Generalmente en el número anterior a salir la versión reducida, se cambia la tipografía y se maqueta pensando que hay que imprimirla a dos tamaños: $100 \%$ y $70 \%$. Hay algunas revistas que no hacen esta doble tirada y que sólo lanzan la versión pocket de la revista, como es el caso de Glamour, donde la revista se diseña y tiene un volumen de paginación mayor y exclusivo en este formato.

Varios estudios de mercado han demostrado que la revista en tamaño A4 se considera un objeto externo, muy incómodo de llevar. Algunas lectoras lo equiparan a la pesadez de llevar un libro, algo externo para dejar sobre una mesa. En cambio, dichos estudios señalan el tamaño pocket como algo portátil que cabe en un bolso, en la cartera e incluso lo equiparan con la portabilidad de los teléfonos móviles. Según un estudio de marketing del Grupo Zeta, algunos de los valores que las lectoras dan a este formato son: manejable, fácil de transportar, mejor precio, más cercano emocionalmente ya que lo llevan encima, es más llamativa, más bonita. El formato grande lo asocian con una mayor madurez, más aspiracional, menos manejable... La desventaja del formato mini estaría en que presenta, además, imágenes más pequeñas ${ }^{2}$.

\subsection{Ahorro de costes}

La reducción del tamaño lleva consigo una reducción de costes de fabricación de entre un 30 y un $50 \%$ incluyendo el ahorro de papel, que cada vez más va incrementando su precio. Ese ahorro, en tiradas de más de 300.000 ejemplares, es un ahorro considerable. Si el coste de fabricación de un ejemplar de la revista Elle es de un euro por ejemplar (con 300 páginas de papel de calidad), el coste de la versión reducida es de 0,5 o 0,6 céntimos de euro por ejemplar.

En el caso de Ragazza Pocket, el departamento de producción ha conseguido:

1. Reducir el coste de fabricación en un $38 \%$ en la edición de bolsillo con relación a la edición normal (de $0,60 €$ a $0,37 €$ en términos de coste marginal).

\footnotetext{
2 Entrevista personal a Oscar Becerra, director de Marketing y Expansión de la Revista del Grupo Zeta, mantenida el 9 de junio de 2006 sobre un informe elaborado por la propia empresa sobre petit-press.
} 
2. Utilizar el mismo papel (couché 2c Lumiblade/Graphocote) y el mismo tamaño de bobina (91 y $141 \mathrm{~cm}$ ) para las dos ediciones.

3. Tirar las dos ediciones en los talleres de la misma imprenta y en la misma máquina (Lithoman).

Para llegar a esto ha sido necesario que la edición de bolsillo sea exactamente una reducción proporcional del $74 \%$ de la edición clásica y cambiar la estructura por cuadernos de la revista ${ }^{3}$.

\subsection{Abaratamiento del precio}

Esta reducción de los costes lleva consigo un abaratamiento significativo del precio de la revista con la misma calidad de papel, fotografía, etc. Tal abaratamiento supone en el mercado prácticamente la venta al $50 \%$ del precio del ejemplar más grande de la revista. Las revistas Premium, que generalmente van acompañadas de promociones, alcanzan un precio de venta al público de entre 3 y 4 euros. Son revistas que al llevar regalo se encarecen, pero el regalo interesa a mucha gente, y les sale rentable económicamente comprar la revista. Pero a la gente que no le interesan las promociones, que sólo están interesadas en el contenido de la revista, se les penaliza.

Por eso, esta versión reducida de la misma tiene todo lo que ellos quieren y a un precio muy reducido. Además salen generalmente (aunque Glamour está empezando a incluir acciones especiales de publicidad) sin regalos, sin manipulado, no lleva encartes publicitarios y a un precio que va desde uno a dos euros.

\subsection{Incremento de las ventas}

Está demostrado que las ventas de las revistas en este formato se incrementan en un $20-30 \%$. Un ejemplo de esto es el de la revista juvenil del grupo Zeta, You. A punto de cerrarla en diciembre de 2005, con una difusión escasa de 30.000 ejemplares, la sacaron en versión pocket sin ninguna promoción y a un precio muy reducido. El cambio fue espectacular, consiguiendo doblar la tirada y que la revista siga a día de hoy en el mercado 4 .

El comentario de funcionan muy bien en ventas es el más escuchado entre las diversas personas contactadas para escribir este artículo. Como si fuera la tabla de salvación de muchas para sobrevivir, se acogen a este formato. Ahora bien: ¿Es el pequeño formato la solución para todos los problemas? Las experiencias nos demuestran que el pequeño formato no es la solución a las penas en el punto de venta. Ha habido fracasos como el de YeS ("Young et Sexy") en Italia, lanzada en pequeño formato y suspendida a los pocos meses. GQ UK ha probado del mismo modo el lanzamiento de un formato mini con muy malos resultados de venta.

\footnotetext{
3 Información facilitada por Oscar Becerra, director de Marketing y Expansión del Grupo Zeta.

4 Información facilitada por Oscar Becerra, director de Marketing y Expansión del Grupo Zeta.
} 
Como siempre, son el contenido, el aspecto visual y el tono los que deben atraer a los compradores. El formato solamente es un elemento más en el marketing mix de una revista; la guinda sobre la tarta de un producto de éxito.

\section{ORIGEN DEL TAMAÑO POCKET EN LAS REVISTAS FEMENINAS. LA MODA NACE EN ITALIA}

Si bien es cierto que es en Estados Unidos, donde la revista apareció en 1939, se mantiene en tamaño convencional ya que es un éxito tal y como está, la propuesta ha sido diferente en Europa. Será en 1997 cuando Condé Nast Italia echa el anzuelo a sus lectores ofreciéndoles también una versión en formato de bolsillo de sus principales cabeceras. El éxito se produjo con los números de diciembre de Vogue y de Ad que adjuntaban al primero el formato reducido del segundo, con el primer número de prueba del nuevo Traveller y con el femenino Glamour en una operación promocional organizada con la empresa de cosmética Clinique. gen:

El director de marketing de la empresa editora, Massimo Perrino, explica su ori-

La idea nació a finales de 1996, a partir de una sencilla constatación: en un panorama más bien desolador para el mercado de las publicaciones femeninas italianas, que de 1994 a 1997 habían perdido en conjunto el 18\% de las ventas en quioscos y el $15 \%$ de las suscripciones, Glamour es la única que había experimentado una tendencia positiva, pasando de 71.000 a 132.000 ejemplares vendidos y duplicando las páginas de publicidad. La elección del formato de bolsillo quería ser un ulterior signo de distinción y, junto con la reducción de precio de portada a 2.500 liras, debería permitir que se llegue a una difusión de 200.000 ejemplares (II Grande Libro Della estampa italiana, 1998: 277,126).

Las reacciones fueron siempre positivas y el propio Glamour, lanzado en 1992 y dirigido por Valeia Corbetta, fue la cabecera elegida para el paso definitivo al formato de bolsillo: reducido al $65 \%$ respecto al tradicional. La decisión se tomó después de una larga investigación de marketing que comprendió también la venta en algunas áreas de prueba del formato de bolsillo de marzo a julio de ese mismo año. El Consejero Delegado de Condé Nast en Italia, Gianpaolo Grandi, decidió hacer una prueba con esta revista publicándola en pequeño formato en las regiones de Umbría y Calabria.

Según las intenciones de la empresa Condé Nast, la operación intentó atraer a nuevas lectoras intrigadas por el nuevo formato y animadas por el bajo precio de portada. El mensual fue respaldado también por una campaña publicitaria de tres meses y 2,5 millardos: uno destinado a la promoción en el punto de venta con los oportunos expositores, los otros divididos entre prensa y radio.

La amplia investigación para la elección del formato reducido, realizada por la empresa Research International, se desarrolló en más fases. Inicialmente se analizaron los contenidos de todas las publicaciones femeninas italianas. Después Glamour, revisado en materia gráfica y en la parte de las rúbricas, se sometió a una investigación cualitativa en la que se venía presentando también el formato reducido. Posteriormente se inició la venta en dos áreas de prueba: en una sólo había Glamour en 
tamaño reducido en los quioscos a 2.500 liras; en la otra estaban los dos formatos a precios diferenciados (5.000 liras el tradicional). Perrino recuerda que las ventas se triplicaron sólo en el área con él y en la otra se duplicaron. Para completar el cuadro, en una tercera área de prueba se puso a la venta el formato de bolsillo a 5.000 liras: en el primer mes las ventas han aumentado un $50 \%$, en el segundo un $100 \%$ (II Grande Libro Della estampa italiana, 1998: 277, 126).

La última comprobación fue una encuesta cuantitativa en doble visita: las compradoras de Glamour en los quioscos eran entrevistadas en ese mismo momento y a continuación en casa con una charla prolongada. El temor era que les atrajera más que nada la novedad y que este tipo de formato agradara sobre todo a las más jóvenes, explica Perrino. Pero las respuestas de las lectoras pusieron en el primer lugar la practicidad del formato de bolsillo y la edad media de las compradoras ha resultado ser 32 años, perfectamente en línea con el actual target de Glamour (II Grande Libro Della estampa italiana, 1998: 277, 126).

Los resultados totales de la prueba fueron asombrosos: con el formato mini, las ventas aumentaron una media de un $60 \%$. Así, el primer número del pequeño Glamour salió en octubre de 1998 pero convivirá con la versión estándar hasta enero de 2000 donde ésta desaparece definitivamente.

Hoy en día y como se puede observar en la tabla 1, Glamour Italia vende en torno a los 300.000 ejemplares. En 1997 su difusión no rebasaba los 150.000 ejemplares. Los ingresos en concepto de publicidad se han multiplicado por dos durante este período. El éxito no ha tardado en copiarse. Tres revistas italianas han adoptado el mismo formato: Cipria, una revista femenina de gama alta, Al Volante, una revista mensual del automóvil, y Quark, el último lanzamiento de Hachette Rusconi. En 2001, Mondadori había lanzado Cambio, un competidor de Al Volante igualmente en formato mini.

Tabla 1. Formato Premiun y Pocket de Glamour Italia.

\begin{tabular}{lll}
\hline Glamour italia & Formato Premiun & Formato Pocket \\
\hline $\begin{array}{l}\text { Año de salida al } \\
\text { mercado }\end{array}$ & $1992-2000$ & 1998 \\
Precio & & \\
Medidas & 5.000 liras & 2.500 liras \\
Tirada & $206 \times 275 \mathrm{~mm}$ & $167 \times 223 \mathrm{~mm}$ \\
\hline Difusión & 196.065 (nov.97/oct98) & 352.205 (abril04/marzo05) \\
\hline
\end{tabular}

Fuente: Elaboración propia.

\section{EL TAMAÑO POCKET EN EUROPA. UN CASO ÚNICO: GLAMOUR UK}

Inspirado por el éxito de Glamour en Italia, Condé Nast lanzó Vogue Business en Alemania en pequeño formato en 1999. Un año después, Condé Nast tomó la decisión de lanzar Glamour, que ahora vende más de 482.434 ejemplares por mes, lo que le ha situado como líder en difusión de revistas femeninas de gama alta. 
Axel Springer reaccionó lanzando un formato pocket de su revista Allegra al precio de 1,80€, conservando siempre el formato tradicional a 2,60 €. A pesar del lanzamiento de Glamour, un competidor directo en la banda de mujeres de 25-35 años, su difusión ha saltado hasta los 291.469 ejemplares, una progresión del 33\% con relación al año 2000.

En marzo de 2001, Condé Nast UK lanzó Glamour en tamaño para el bolso. Dudando de que los éxitos alcanzados en Italia y Alemania se pudieran repetir en el Reino Unido, entrevistaron a 2.000 mujeres antes del lanzamiento: el 98\% encontraron el formato muy atractivo.

EMAP UK también redujo el formato de las revistas Top Santé y Bliss. La directora de marketing de EMAP aseguraba recientemente que las ventas de Bliss habían progresado en más del $10 \%$ después de haber reducido el formato y doblado el paginado.

Hearst ha lanzado ya la edición mini de Cosmopolitan en Francia, en España y en Grecia. Condé Nast también sacó en 2002 la versión española de Glamour en pequeño formato. Hello! y Family Circle en Inglaterra también han estudiado la posibilidad de reducir su formato. Pacific Publications acaba de relanzar su revista femenina $B$ en Australia en tamaño de bolsillo.

Axel Springer en Francia lanzó en 2002 la mensual femenina Bien dans ma Vie en formato A5, que ha encontrado su espacio en un segmento de mercado muy competitivo, alcanzado una difusión media superior a los 180.000 ejemplares. En cuanto a Cosmopolitan y Jalouse, que se venden en dos formatos diferentes, los resultados de ventas no son excepcionales.

Por su parte, Condé Nast lanzó Glamour en Francia en pequeño formato en 2004 siguiendo las directrices del Consejero Delegado de Condé Nast France, Didier Suberbielle, que declaró en Figaro Economie que Hay por parte del accionariado la voluntad de dar a la filial francesa un nuevo impulso [..... . Primero consideramos lo que funciona en el extranjero y luego tratamos de importar los conceptos adaptables al mercado francés (II Grande Libro Della estampa italiana, 1997: 261, 24).

También Psychologies y Vivre au féminin han pasado a este formato y la nueva Esprit Femme con su primer número pocket ha llegado a los 200.000 ejemplares vendidos.

\subsection{Un caso único: Glamour UK}

Sin duda alguna, el éxito más espectacular entre los pequeños formatos ha sido el de Condé Nast con Glamour, especialmente en Inglaterra (junio de 2000), donde se ha convertido en la revista más vendida del Reino Unido. Estudiemos más de cerca los factores clave del éxito de Glamour UK.

\subsubsection{El formato}

Cuando Condé Nast lanzó Glamour en formato A5, algunos competidores afirmaron que eso jamás iba a marchar bien en el Reino Unido. En especial, porque 
para los anunciantes lo grande siempre es hermoso. Pero Nicholas Coleridge, el Consejero Delegado y Director Gerente de Condé Nast, aseguró que, en los estudios de mercado previos, el $90 \%$ de las lectoras objetivo habían encontrado cómodo y atractivo el formato y que las mujeres modernas que toman el metro, el autobús, que van del trabajo al gimnasio y del gimnasio al restaurante, van a apreciar mucho un formato así de manejable (II Grande Libro Della estampa italiana, 1998: 277, 163).

Jo Elvin, directora de redacción de Glamour, fue todavía más lejos: Nosotras, las mujeres, tenemos una relación emocional con las cosas que metemos en nuestros bolsos. Eso es el centro de nuestra vida (II Grande Libro Della estampa italiana, 1998: 277, 163). De hecho, el eslogan de la campaña publicitaria de lanzamiento de Glamour en el Reino Unido y en España ha sido la revista que cabe en tu bolso al igual que en tu vida.

Pero cuando se habla de formato, suele olvidarse una de las dimensiones de una revista: el grosor. Y los números de Glamour nunca tienen menos de 300 páginas en total en todas las ediciones europeas. Así pues, se puede deducir que el grosor constituye una parte esencial de la fórmula Glamour.

Se eligió un papel más barato, pero de buena calidad y se aligeró el peso de cada página eligiendo papel de $70 \mathrm{gr}$. en vez del de $80 \mathrm{gr}$. de otros títulos. Aunque el volumen de páginas era grande, el tamaño de la revista y el papel contribuían a dar sensación de ligereza y de portabilidad. Además necesitaban un papel moderno, así que se tuvo en cuenta que los lectores británicos prefieren un color de papel azul o Persil blanco mientras que en Alemania o Italia tienden a preferir uno más cremoso, amarillo o blanco.

\subsubsection{El precio}

Sin duda, es uno de los factores clave del éxito de Glamour. Con un precio de $1,80 €$ (precio de lanzamiento 1,50 €), Glamour es una libra esterlina más barata por término medio que sus competidoras, por ejemplo, Cosmopolitan (2,80 €). Su precio es incluso competitivo con el de las revistas semanales populares de gama alta como Hello! (1,60 €). En España han seguido la misma estrategia: el precio de la revista $(1,50 €)$ es más bajo que el de los semanarios populares $(1,70 €)$ y exactamente la mitad de los femeninos de moda $(3,00 €)$.

\subsubsection{La campaña de lanzamiento}

La campaña promocional de Glamour en Inglaterra ha sido la más cara en 11 años, después del lanzamiento de Marie Claire en 1990. El presupuesto de lanzamiento rebasaba los 8 millones de euros (53 millones de francos), una partida importante de los cuales fue destinada para los expositores enviados a los cerca de 30.000 puntos de venta.

La campaña de medios, concebida por la agencia J. Walter Thompson Label, usaba un enfoque humorístico de la vida cotidiana de las mujeres jóvenes para desarrollar una simpatía entre la marca y el objetivo. El mensaje de fondo era muy comprensivo, muy moderno: una mujer está llena de contradicciones, pero eso es curioso; se hacen las mismas tonterías, no hace falta romperse la cabeza por eso. 
El eslogan era the magazine that fits in your bag and in your life (la revista que encaja en tu bolso y en tu vida).

\subsubsection{La elección de contenidos}

Nada revolucionario: moda, belleza, gente, psicología, salud, nutrición, sexo, pareja, trabajo, cartelera de espectáculos, correo de lectores (cartas al/a la directora/a): A excepción de una visión más holística de la mujer y de una aceptación de sus contradicciones vitales. Efectivamente, la imagen que las mujeres tienen de sí mismas parece haber evolucionado mucho. Que las mujeres sean contradictorias no tiene nada de nuevo... La novedad es que ellas lo acepten... ;Y que se rían de ello! La promesa de Glamour es procurar que las mujeres se sientan bien en su piel a pesar de sus debilidades. Es ésta una revista que trata de ayudar a las lectoras a moldear su imagen exterior, pero también a mejorar su bienestar interior. Glamour es una mezcla relajada de lo necio y de lo serio, un artículo sobre tintes de pelo puede estar junto a otro sobre la fertilidad. El fascismo corporal, la normalidad, el esnobismo y la hipersexualidad de otras revistas han desaparecido.

Un humor muy juvenil, muy fresco y muy positivo se irradia desde cada una de las páginas de Glamour UK. De la misma manera que su colega Heat, un semanario popular de enorme éxito, la ironía y la jerga juvenil son los cimientos sobre los que se ha construido la revista. Por el contrario, las revistas que siempre se toman la vida en serio sufren cada vez más, como es el caso de Cosmopolitan, que ha perdido la corona de líder de la prensa femenina inglesa en beneficio de Glamour.

Éste ha sido el lanzamiento más importante de revistas de prensa inglesa en 30 años y ha conseguido ser la líder de las revistas femeninas de gama alta en Inglaterra. Se puede observar en la tabla 2, los altísimos índices de difusión conseguidos en 2004 .

Tabla 2. Formato Pocket de Glamour UK.

\begin{tabular}{ll}
\hline Glamour UK & Formato Pocket \\
\hline Sale al mercado & Junio 2000 \\
Precio & 1,80 euros \\
Medidas & $167 \times 225 \mathrm{~mm}$ \\
Tirada & $700.000(2004)$ \\
Difusión & $585.000(2004)$ \\
\hline
\end{tabular}

Fuente: Elaboración propia.

\section{LAS REVISTAS FEMENINAS ESPAÑOLAS COPIAN LA IDEA}

En septiembre de 1999, Hachette Filipacchi se decide a lanzar en España un formato reducido de su revista juvenil Ragazza: Ragazza Pocket. A partir de ese momento, los dos formatos cohabitan en los puntos de venta, el grande $(2,1 €)$ era el 
único que ofrecía productos adicionales. El pequeño formato $(1,50 €)$, impreso siempre en tirada controlada (60.000 ejemplares; con un objetivo de tasa de invendidos del $20 \%$ ) se lanzó con un presupuesto de promoción muy reducido como un formato de moda únicamente a través de la colocación de producto en las telenovelas jóvenes ${ }^{5}$.

En la tabla 3 se muestran los datos comparativos de tirada y difusión de los dos formatos. Como se puede observar, aunque las cifras del pocket son inferiores, les permiten vender más revistas y no perder cuota de mercado.

Tabla 3. Formato Premiun y Pocket de Ragazza.

\begin{tabular}{lll}
\hline Ragazza & Fom ato Premiun & Formato Pocket \\
\hline Sale al mercado & Noviembre 1989 & Septiembre 1999 \\
Precio & 2,50 euros & 1,00 euros \\
Medidas & $228 \times 297 \mathrm{~mm}$. & $177 \times 230 \mathrm{~mm}$ \\
Tirada & 89.608 (O.JD Jul05iJun06) & 63.833 (O.JD Jul05iJun06) \\
& 54.211 (OJD Jul05iJun06) & 41.595 (OJD Jul05iJun06)) \\
\end{tabular}

Fuente: OJD y elaboración propia.

Tras el éxito de esta revista y de ver la repercusión internacional que la revista Glamour estaba teniendo, Cosmopolitan decide lanzar su versión mini en febrero de 2002. El éxito fue arrollador 6 . Este éxito en ventas se puede observar en la tabla 4, llegando casi a los 100.000 ejemplares vendidos en el formato mini.

Tabla 4. Formato Premiun y Pocket de Cosmopolitan.

\begin{tabular}{|c|c|c|c|}
\hline Cosmopolitan & Fomato Premiun & & Formato Pocket \\
\hline Sale al mercado & Octubre 1990 & & Febrero 2002 \\
\hline Precio & 2,60 euros & & 1,80 euros \\
\hline Medidas & $213 \times 277 \mathrm{~mm}$ & & $177 \times 230 \mathrm{~mm}$ \\
\hline \multirow[t]{2}{*}{ Tirada } & 212.789 & (OJD & 133.050 (OJD Jul05/Jun06) \\
\hline & Jul05/Jun06) & & \\
\hline \multirow[t]{2}{*}{ Difusión } & 139.429 & (OJD & 84.935 (O.JD Jul05/Jun06) \\
\hline & Jul05/Jun06) & & \\
\hline
\end{tabular}

Fuente: OJD y elaboración propia.

\footnotetext{
$\overline{5}$ Información facilitada por Oscar Becerra, director de Marketing y Expansión del Grupo Zeta.

${ }^{6}$ Entrevista con Juan Elvira y María Isabel Abad del Dpto. de Maquetación de Cosmopolitan.
} 
Es en octubre de 2002 cuando Conde Nast lanza su versión de Glamour en un mercado tan conservador como el español con una tirada inicial de 800.000 ejemplares y 600.000 vendidos. La revista, como se puede observar en la tabla 5, literalmente arrasa en ventas convirtiéndose desde entonces y hasta la actualidad en la primera revista del segmento en difusión y audiencia.

Tabla 5. Formato Pocket de Glamour España.

\begin{tabular}{ll}
\hline Glamour & Fomato Pocket \\
\hline Sale al mercado & Octubre 2002 \\
Precio & 1,70 euros \\
Medidas & $167 \times 223 \mathrm{~mm}$ \\
Tirada & 416.071 (O.JD Jul05/Jun06) \\
Difusión & 258.544 (O.JD Jul05/Jun06) \\
\hline
\end{tabular}

Fuente: OJD y elaboración propia.

Además Glamour, al pertenecer a un gran grupo editorial como es Condé Nast intercambia material dentro de la misma casa. Tienen acceso a la web de textos de Glamour Italia (edición madre) y luego facturan internamente según el uso de material que hagan de ella. La paginación es altísima, con lo que el contenido es muy amplio y así pretenden llegar a un mayor número de lectoras.

Ante el éxito de este tipo de publicaciones, las demás revistas femeninas no tienen más remedio que adecuarse al mercado. En marzo de 2006 lanzan su formato pocket Woman (con una tirada de 70.000 ejemplares) y Psychologies y en abril del mismo año lo hace Marie Claire aunque todas lo siguen simultaneando con su formato grande o premium. En el caso de Pychologies se lanzan tres promociones: la revista premiun con regalo, premiun sin regalo y la pocket ${ }^{7}$.

En las tablas 6, 7 y 8 se pueden comprobar los últimos datos de ventas de estas últimas revistas en incorporarse a la versión pocket. Aunque de momento no tengan grandes cifras de difusión, es innegable que tienen que estar en el mercado en este formato y no perder cuota ni protagonismo dada la gran competencia que existe entre ellas.

\footnotetext{
7 Entrevista con Mónica Martínez, directora de Marketing Editorial de Woman (6 de junio de 2006) y con Belén Ceda, del Departamento de Maquetación de Marie Claire (27 de mayo de 2006)
} 
Tabla 6. Formato Premiun y Pocket de Woman.

\begin{tabular}{lll}
\hline Woman & Fomato Premiun & Formato Pocket \\
\hline Sale al mercado & Octubre 1992 & Marzo 2006 \\
Precio & 3 euros & 1,90 euros \\
Medidas & $225 \times 297 \mathrm{~mm}$ & $170 \times 223 \mathrm{~mm}$ \\
Tirada & 156.000 (O.JD Jul05/Jun06) & 62.400 (O.JD Jul05/Jun06) \\
Difusión & 106.168 (O.JD Jul05/Jun06)) & 32.136 (OJD Jul05/Jun06) \\
\hline
\end{tabular}

Fuente: OJD y elaboración propia.

Tabla 7. Formato Premiun y Pocket de Psychologies.

\begin{tabular}{lll}
\hline Psychologies & Formato Premiun & Formato Pocket \\
\hline Sale al mercado & Febrero 2005 & Marzo 2006 \\
Precio & 3 euros & 1 euro \\
Medidas & $215 \times 280 \mathrm{mmm}$ & $169 \times 220 \mathrm{~mm}$ \\
Tirada & 230.900 (O.JD Jul05iJun06) & 64.300 (OJD Jul05iJun06) \\
Difusión & 121.013 (O.JD Jul05iJun06) & 28.569 (OJD Jul05iJun06) \\
\hline
\end{tabular}

Fuente: OJD y elaboración propia.

Tabla 8. Formato Premiun y Pocket de Marie Claire.

\begin{tabular}{lll}
\hline Marie Claire & Fomato Premiun & Formato Pocket \\
\hline Sale al mercado & 1987 & Abril 2006 \\
Precio & 3,60 euros & 2 euros \\
Medidas & $227 \times 297 \mathrm{~mm}$ & $176 \times 230 \mathrm{~mm}$ \\
Tirada & 193.000 (O.JD Jul05/Jun06) & 60.333 (O.JD Jul05/Jun06) \\
Difusión & 125.024 (O.JD Jul05/Jun06) & 23.260 (O.JD Jul05/Jun06) \\
\hline
\end{tabular}

Fuente: OJD y elaboración propia.

En febrero de 2007 el grupo editorial Hachette Filipachi decide apostar por el formato pocket para dos de sus buques insignia como Elle y AR. (tablas 9 y 10). La diferencia de este grupo reside en que la distribución de este formato mini con la revista Elle no sólo se hace a través de la venta en quioscos sino que se distribuye con el diario La Razón y con los periódicos del Grupo Joly (Diario de Sevilla, Diario de Cádiz, Europa Sur, Diario de Jerez, Granada Hoy, Málaga Hoy, El Día de Córdoba y Huelva Información) ${ }^{8}$. Así Elle se vende en quioscos o se distribuye bien gratuitamente o con un sobreprecio con los diarios anteriormente señalados. Este dato es

\footnotetext{
${ }^{8}$ Entrevista con María José Martín, responsable de Marketing de Hachette Filipachi (15 de junio de 2007).
} 
importante y habrá que tenerlo en cuenta en la tabla 9 con los datos de tirada y difusión.

Tabla 9. Formato Premiun y Pocket de Elle.

\begin{tabular}{|c|c|c|c|}
\hline Elle & Formato Premiun & & Formato Pocket \\
\hline Sale al mercado & Octubre 1986 & & Febrero 2007 \\
\hline Precio & 3,00 euros & & 1,70 euro \\
\hline Medidas & $228 \times 297 \mathrm{~mm}$ & & $170 \times 220 \mathrm{~mm}$ \\
\hline Tirada & $\begin{array}{l}291.525 \\
\text { Jul05/Jun06) }\end{array}$ & (O.JD & $\begin{array}{l}170.000 \text { (datos intemos. No } \\
\text { hay datos O.JD) }\end{array}$ \\
\hline Difusión & $\begin{array}{l}\text { 209.636 } \\
\text { Jul05/Jun06) }\end{array}$ & (OJD & $\begin{array}{l}145.000 \text { (datos intemos. No } \\
\text { hay datos O.JD) }\end{array}$ \\
\hline
\end{tabular}

Fuente: OJD y elaboración propia.

Tabla 10. Formato Premiun y Pocket de $A R$.

\begin{tabular}{|c|c|c|c|}
\hline$A R$ & Fomato Premiun & & Formato Pocket \\
\hline Sale al mercado & Noviembre 2001 & & Febrero 2007 \\
\hline Precio & 2,50 euros & & 1,70 euro \\
\hline Medidas & $228 \times 297 \mathrm{~mm}$ & & $170 \times 220 \mathrm{~mm}$ \\
\hline Tirada & $\begin{array}{l}248.500 \\
\text { Jul05/Jun06) }\end{array}$ & (O.JD & $\begin{array}{l}165.000 \text { (datos internos. No } \\
\text { hay datos O.JD) }\end{array}$ \\
\hline Difusión & $\begin{array}{l}\text { 134.672(O.JD } \\
\text { Jul05/Jun06) }\end{array}$ & & $\begin{array}{l}145.000 \text { (datos internos. No } \\
\text { hay datos OJD) }\end{array}$ \\
\hline
\end{tabular}

Fuente: OJD y elaboración propia.

Nos encontramos con revistas de alta gama a un precio realmente asequible, que no llega a los dos euros. La reducción de tamaño y remodelación de páginas que este nuevo formato conlleva supone tal ahorro de papel que se puede sacar la revista al mercado a un precio realmente competitivo. La gama de revistas que hay en el mercado por este precio y que pueden tener a estas lectoras como potenciales clientas es muy amplia. Las más cercanas al target de lectoras de las revistas femeninas de alta gama son las revistas femeninas prácticas, las revistas de salud e incluso la prensa del corazón.

Así, la lectora que acostumbraba a comprar una revista femenina de corte práctico, esto es: Mía (1,10 euros), Clara (1,80 euros), Única (1 euro), puede decantarse ahora prácticamente por el mismo precio por una revista de alta gama; es decir de más calidad pero al mismo precio.

Además el creciente éxito de las revistas de salud dedicadas a la mujer también las convierte en potenciales competidoras de las femeninas de alta gama, que casi siempre incluyen temas relacionados con el bienestar y la salud. Ahora, con este ajuste de precios que conlleva la miniaturización, se pueden comprar por el mismo 
precio una de alta gama que una de salud; Vive (1,90 euros), Línea (1,50 euros), Vivir Mejor (1 euro).

En cuanto a las revistas del corazón, también hay un hueco de mercado dispuesto a comprar una de alta gama pocket en vez de una de corazón (eso sí, por el mismo precio). Tenemos, por ejemplo, Hola (1,80 euros) y Lecturas (1,90 euros). Incluso los nuevos lanzamientos dedicados a la mujer como Bulevar ( 1,80 euros), no sobrepasan la frontera de este precio.

\section{CONCLUSIONES}

Los esfuerzos que hacen los distintos grupos editoriales por investigar y desarroIlar nuevas propuestas acordes tanto a los anunciantes como a los lectores, llevan a auténticos descubrimientos de formato editorial y publicitario como este formato de bolsillo.

Ante un panorama para la prensa femenina de tamaño tradicional cada vez más negro (las ventas de revistas siguen cayendo y cada vez está más en entredicho el fenómeno promocional) la versión pocket de las mismas se convierte en una estrategia de las editoriales para captar a un público más joven con menor poder adquisitivo y ofreciendo la misma calidad de producto. Las revistas femeninas han ido siguiendo el crecimiento de edad de sus lectoras, de modo que ha quedado un hueco libre de mujeres más jóvenes (entre 23 y 29 años) que constituyen su principal objetivo.

Tanto las femeninas de alta gama, como las revistas prácticas pueden abarrotar los quioscos en breve en formato pocket, ya no hay ninguna razón para que no lo hagan. Aunque este formato no tiene asegurada la llave del éxito, lo que sí es cierto es que la oferta del mercado presenta este nuevo formato, que las ventas funcionan realmente bien y que no adecuarse al mismo significa aislarse. Hay algunas publicaciones españolas reticentes como Vogue, pero es sólo cuestión de tiempo que pasen al formato pequeño. Estas mismas publicaciones eran también a comienzos de los 90 reticentes a las promociones y ahora es absolutamente impensable una revista $\sin$ ellas $^{9}$.

Además, el nuevo mercado potencial de lectoras que parece vislumbrarse con este nuevo formato es enorme. Pueden acceder a nuevos targets e invadir otros sectores de revistas como el práctico, salud e incluso corazón. No sólo el tamaño, sino el precio realmente competitivo se convierte en un factor fundamental. Así pues, se ofrece alta gama (alta calidad fotográfica, tipográfica, firmas de renombre, modelos...) a un precio muy asequible.

Los últimos lanzamientos en versión pocket han sido El Mueble (septiembre 2006), AR y Elle (junio 2007) y Telva (octubre 2007). Ya sólo queda esperar para ver si la nueva versión española de la americana Vanity Fair se hará en formato grande o pocket.

\footnotetext{
${ }^{9}$ Entrevista con Samari Fernández Feito de Telva y con María José Martín del Departamento de Marketing de Elle (12 de junio de 2006).
} 


\section{REFERENCIAS BIBLIOGRÁFICAS}

ADDICOTT, R. "The incredible shrinking Magazine reducing size of magazines". The Magzine for Magazine Management, $\mathrm{n}^{\circ}$ 1,(2002), pp. 46-53.

ALBERT, P. La presse francaise. París: La documentation francaise, 2004.

ARENAS, L. "Glamour: el mundo en un bolso". IPMARK, n 435, (diciembre, 2003), pp.167-178.

CAÑO, J. Revistas: una historia de amor y un decálogo. Madrid: Eresma \& Celeste Ediciones, 1999.

CONTROL. "Debate revistas: hablamos de lanzamientos de marketing editorial", $\mathrm{n}^{\circ}$ 520, (2006), pp. 26-28.

GOUGHT-YATES, A. Understanding Women's Magazines. London: Routledge, 2003.

HERMES, J. Reading Women's Magazines. London: Polity Press, 1995.

MEDINAVEITIA, E. "El año del glamour”. El Publicista, n 91, (Octubre 2003), p. 52.

MONTERO, F. Marketing de periódicos y revistas. Madrid: Pirámide, 2005.

PALACIOS, F. "Revista AR. Para mujeres de más de 30". IPMARK, n 593, (Diciembre 2002), p. 55.

PLAZA, J. Modelos de varón y mujer en las revistas femeninas para adolescentes. Madrid: Fundamentos, 2005.

SAEZ, M. "Las claves del Glamour". Anuncios, n 587, (Noviembre 2002), p. 22.

- (1995) "Cosmoadictas". Anuncios, nº 670, (Octubre), pp.36-38.

"Especial revistas. Un ejercicio digno". Anuncios, n 95, (Diciembre 2001), pp.68-69.

"Glamour". II Grande Libro della estampa Italiana, n² 260, (II-1997), p. 24.

"Glamour”. II Grande Libro della estampa Italiana “, n 271, (II-1998), p. 24.

“Glamour". II Grande Libro della estampa Italiana, n 277, (IX-1998), p. 126.

"Glamour". II Grande Libro della estampa Italiana, n 305, (III-2002), p.35.

"Glamour". II Grande Libro della estampa Italiana, n 327, (V-2003), p. 36.

"Glamour". II Grande Libro della estampa Italiana, n 340, (II- 2004), p.45. 
"Glamour". II Grande Libro della estampa Italiana, n 362, (V- 2006), p.134.

"Glamour sceglie il formato pocket". Prima della Communicazione, $\mathrm{n}^{\circ} 277$, (1998), pp.57-59.

“Informe revistas 2006: más calidad, menos cantidad". IPMARK, nº654, (2006), p.46.

"Las claves de Glamour". Anuncios, n 987, (Diciembre 2002), pp.56-57.

"Las revistas de consumo aumentan sus ingresos pero sufren un retroceso en la publicidad". Noticias de la Comunicación, nº 260, (Febrero 2006), pp.68-79.

"Revistas 2006. Más calidad, menos cantidad". IPMARK, n 655, (Febrero 2006), p.46.

\section{Breve semblanza de la autora}

María Ganzabal Learreta es doctora en periodismo y profesora desde 2003 del Departamento de Comunicación Audiovisual y Publicidad de la Universidad del País Vasco. Ha trabajado en la revista femenina New Woman Magazine en Nueva York y ha escrito numerosos artículos sobre prensa femenina y comunicación. Actualmente coordina e imparte clases en varios masteres de la Universidad del País Vasco.

(Recibido el 24-10-07, aceptado el 31-03-08) 